\title{
Advances in wearable high-density diffuse optical tomography: first applications of a new commercial technology and development of an infant-specific research device
}

Zhao, Hubin, Brigadoi, Sabrina, Hill, Reuben, Smith, Greg, Dunne, Luke, et al.

Hubin Zhao, Sabrina Brigadoi, Reuben Hill, Greg Smith, Luke Dunne, Elisabetta Maria Frijia, Samuel Powell, Nick Everdell, Robert J. Cooper, "Advances in wearable high-density diffuse optical tomography: first applications of a new commercial technology and development of an infantspecific research device

," Proc. SPIE 11074, Diffuse Optical Spectroscopy and Imaging VII, 1107403 (11 July 2019); doi: 10.1117/12.2526976 


\title{
Advances in wearable high-density diffuse optical tomography: first applications of a new commercial technology and development of an infant-specific research device.
}

\author{
Hubin Zhao ${ }^{\text {a }}$, Sabrina Brigadoi ${ }^{b}$, Reuben Hillc, Greg Smith ${ }^{c}$, Luke Dunnec, Elisabetta Maria Frijia ${ }^{\text {a }}$, \\ Samuel Powell' ${ }^{\mathbf{c}}$ Nick Everdell ${ }^{\mathbf{c}}$ and Robert J Cooper \\ ${ }^{a}$ DOT-HUB, Biomedical Optics Research Laboratory, Department of Medical Physics and Biomedical Engineering, \\ University College London, London WC1E 6BT, UK \\ ${ }^{b}$ Department of Developmental Psychology, University of Padova, Padova, Italy \\ ${ }^{c}$ Gowerlabs Ltd., London, UK
}

\begin{abstract}
We discuss advances in and applications of fibre-less, wearable, high-density diffuse optical tomography technologies, including a new device specifically for the newborn infant that employs flex-rigid PCB technology and provides channel density approaching 10 channels $/ \mathrm{cm}^{2}$.
\end{abstract}

OCIS codes: (170.6960) Tomography; (170.0110) Imaging systems; (230.0250) Optoelectronics.

\section{Introduction}

In diffuse optical tomography (DOT), depth-resolved images of the changes in concentration of the oxygenated and deoxygenated forms of haemoglobin are obtained using an array of sources and detectors of near-infrared light arranged to provide multiple source-detector separations and overlapping sensitivity profiles [1]. While DOT has numerous advantages (it is non-invasive, non-ionizing, inexpensive and well tolerated by a wide range of subjects) its uptake as a functional neuroimaging method has been limited by several fundamental challenges. These challenges include its traditionally limited resolution and the sensitivity of diffuse optical methods to haemodynamics in the superficial tissues (particularly the scalp) [2], which can corrupt measurements of the brain.

In recent years, a range of studies have demonstrated that increasing the sampling density of DOT methods significantly improves both their resolution and their ability to separate scalp and brain haemodynamics [3], [4]. However, the continued use of optical fibre bundles limits how far researchers have been able to push high-density DOT methodologies. The size and weight of optical fibre bundles restricts how many sensors can be applied to the scalp, which in turn limits sampling density and/or field-of-view. Increasing the number of optical fibre bundles also quickly undermines one of the key advantages of DOT; i.e. that the technique is highly portable.

The ability to image human brain function using a wearable device has long been a goal of near-infrared spectroscopy (NIRS) and diffuse optical tomography research, and several wearable diffuse optical technologies are already commercially available. However, these technologies are either cumbersome, have a limited field of view, or provide insufficient channel number and density to perform effectively as imaging systems.

In late 2016, we presented the first functional images of the human brain obtained using a fibreless, high-density DOT system known as the micro-NTS ( $\mu$ NTS)[5]. A year later at ECBO 2017, we described a 12-module version of this device, which had a sufficiently large field-of-view to cover both left and right sensorimotor regions of the brain. Since that time, our commercial collaborators Gowerlabs Ltd. have taken significant steps in the development of this technology to produce the first commercial modular high-density DOT device ('LUMO'). We discuss the preliminary applications of this system below.

In a continuation of this partnership, at we are also developing a version of this technology specifically designed for the vulnerable newborn and pre-term infant. A device that is ideally suited to this cohort must meet certain requirements because of the high curvature of the head and the sensitivity of the infant scalp: the device must be ultralight-weight, have a low-profile to help minimize motion artifacts and have the capacity to maintain good optical contact with the scalp across the highly curved surface of the infant

\section{Modular HD-DOT technologies}

\subsection{System evolution}

The $\mu$ NTS that was first demonstrated in 2016 consisted of modules based on a $30 \times 30 \mathrm{~mm}$ printed circuit board (PCB), on which were mounted two dual-wavelength LED sources and four photodiode detectors [5]. Subsequent upgrades of this device provided improved EM shielding and added a motion sensor (Invensense inc. USA), enabling 3-axis accelerometry and 3-axis gyroscope data to be obtained from each module. A 12-module version of this device was constructed, and has been used to study the adult motor system during complex movement tasks (in preparation).

Diffuse Optical Spectroscopy and Imaging VII, edited by Hamid Dehghani, Heidrun Wabnitz, Proc. of SPIE-OSA Vol. 11074, 1107403 · C 2019 SPIE-OSA · CCC code: 1605-7422/19/\$21 · doi: 10.1117/12.2526976 

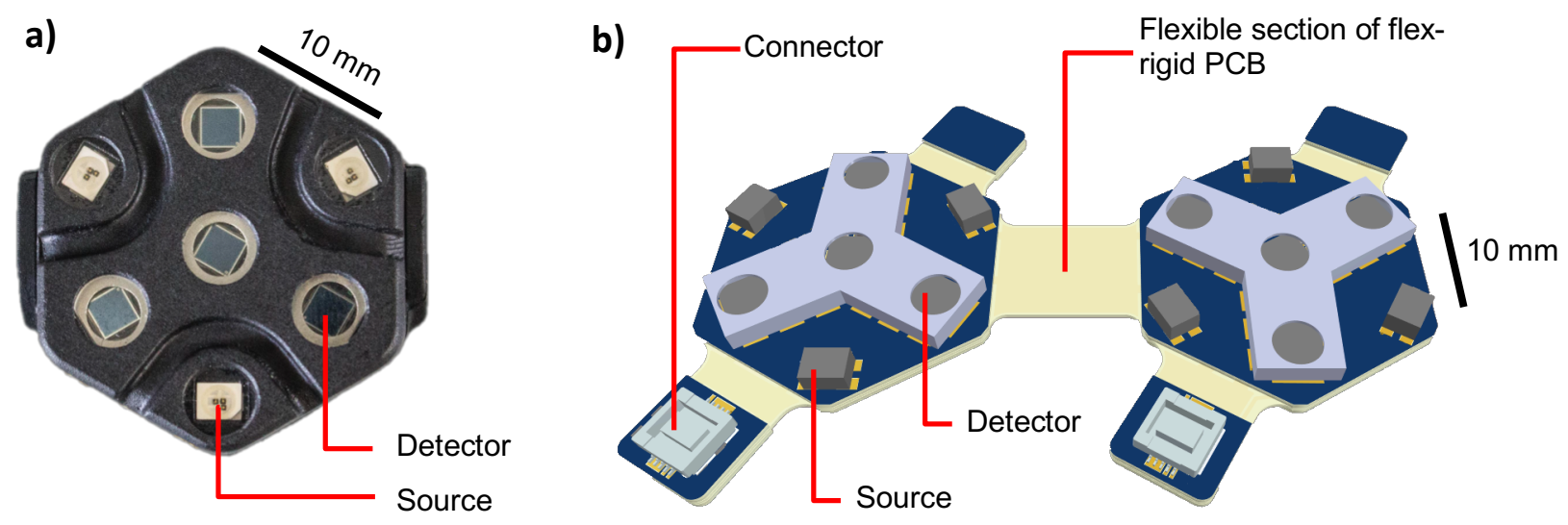

c)

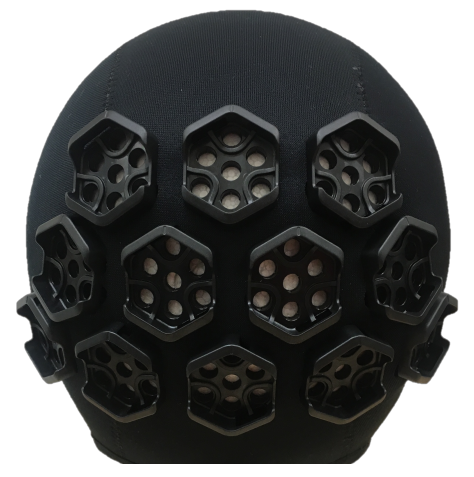

f)
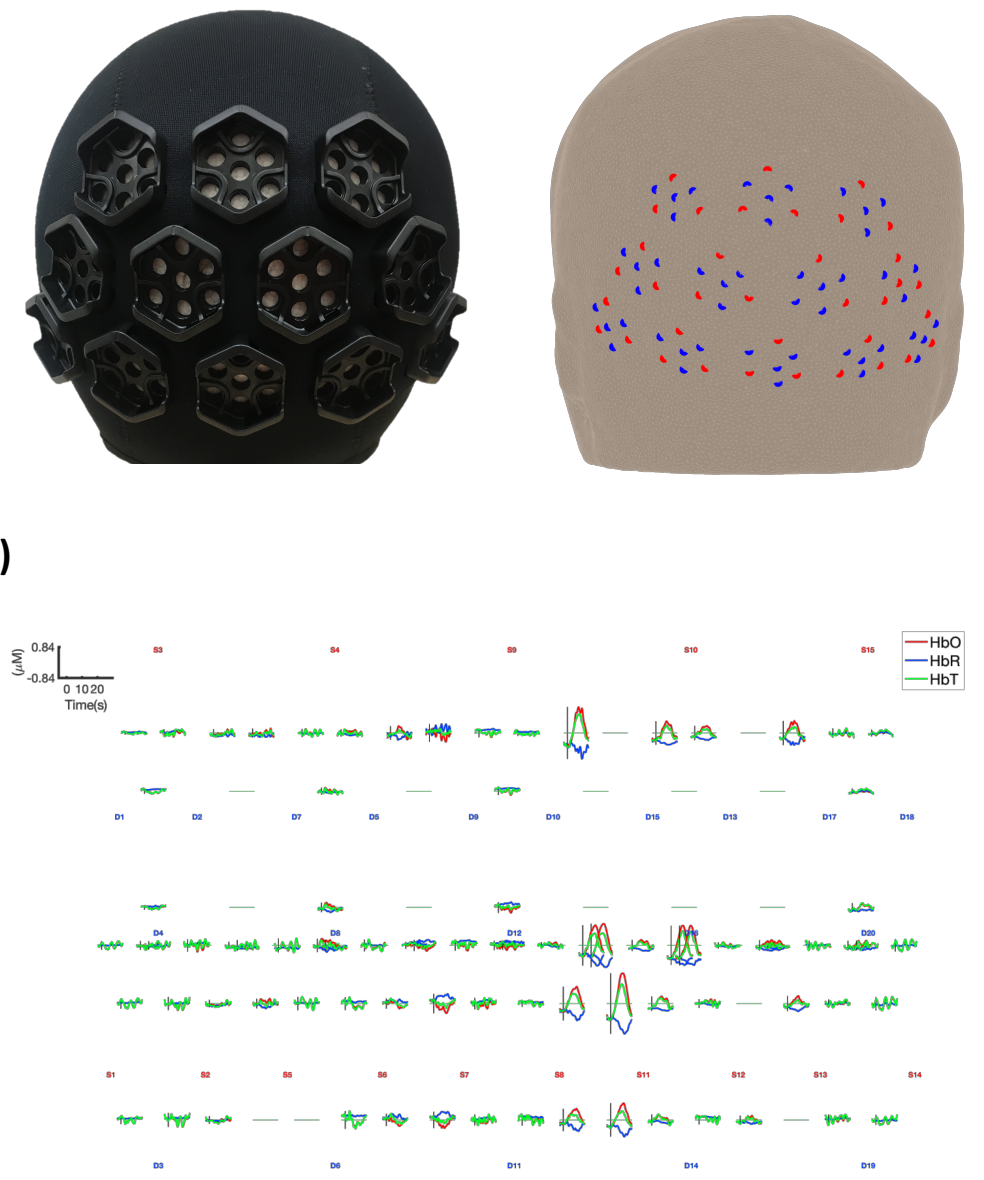

e)

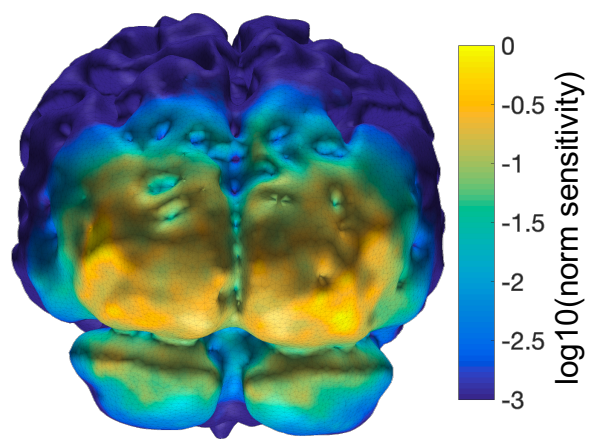

g)

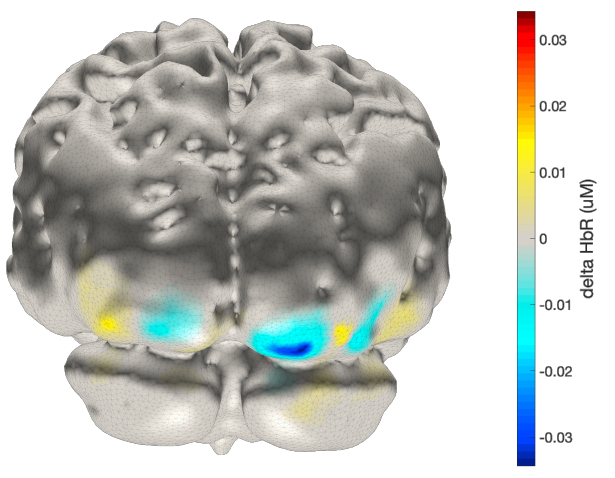

Figure 1. Figure a) shows a prototype of the Gowerlabs' LUMO module, which encapsulates 3 dual-wavelength sources and 4 detectors into a compact hexagonal module. Figure b) shows a diagram of the optical side of the infant-specific ANIMATE dualhex module, which provides 5 sources and 8 detectors. Flexible sections of the board design (shown in light yellow) allow dualhex and triple-hex modules to be daisy-chained together to form a range of flexible HD-DOT arrays. Figures $c, d$ and e show respectively the mechanical set up (with docks); scalp optode locations and cortical sensitivity map of a LUMO array arranged over the visual cortex. Figure f) shows the haemodynamic response to left hemi-field visual stimulus and figure g) shows a preliminary reconstruction of the deoxyhaemoglobin component of this response. 
The recently developed Gowerlabs device ('LUMO') consists of re-designed hexagonal modules (Fig. 1a), each housing 3 dual-wavelength LED sources and 4 photodiode detectors. These modules can be placed into 'docks' (Fig 1c) positioned within a neoprene cap. Any module can be placed in any dock, such that an fNIRS or DOT array can be created to suit the experimental paradigm. Each module provides within-tile source-detector separations of 10 and $20 \mathrm{~mm}$, while inter-tile measurements can provide channels from $20 \mathrm{~mm}$ to $>40 \mathrm{~mm}$ (depending on the subject).

Our new infant-specific device (the ANIMATE system) builds upon the technology developed for the LUMO device. It maintains the same fundamental design of a hexagonal module containing 3 dual-wavelength sources and 4 detectors, but by employing a 10-layer flex-rigid PCB construction, we have been able to minimise the casing requirements and decrease the weight and vertical profile of the on-head components. The ANIMATE system is built around dual-hex (Fig 1b) and triple-hex modules that consist of two and three hexagonal modules (respectively) permanently connected together via flexible sections of the PCB. Both dual-hex and triple-hex modules incorporate male and female board-to-board connectors, and as such can be connected together to form a wide range of array shapes and sizes, while improving the sampling density and maintaining the flexibility necessary to conform to the infant scalp.

\section{Preliminary applications}

We have begun a series of applications of the LUMO device in adults using basic primary functional stimuli such as a finger-stimulation and a visual checker-board. The stimulus was arranged in a block design, with 15 blocks of $15 \mathrm{~s}$ of left or right field stimulation by reversing checkerboard interspersed with a pseudo-random inter-stimulus interval of between 17.5 and $22.5 \mathrm{~s}$. We are also investigating resting-state HD-DOT in the healthy adult.

\section{Results}

Preliminary channel-wise results from one subject (Figure 1f) demonstrate well-localised classical haemodynamic response functions consisting of an increase in oxyhaemoglobin $(\mathrm{HbO})$ and decrease in deoxyhaemoglobin $(\mathrm{HbR})$ in response to left hemi-field visual cortex stimulation. A preliminary reconstruction of the deoxyhaemoglobin response image is shown in figure $1 \mathrm{~g}$. (Note that reconstruction was performed using a subject-specific MRI template and the TOAST++ FEM software package [6]).

\section{Future work}

Functional neuroimaging studies of healthy adults using the LUMO system are ongoing. We are simultaneously beginning a study of 6-month-old infants using a well known social stimulus paradigm to test the suitability of this technology for the infant [7]. The ANIMATE system modules are in the final stages of production, and the device is expected to undergo preliminary on-bench testing in the next 3-6 months.

\section{References}

[1] S. R. Arridge, "Methods in diffuse optical imaging," Philos. Trans. R. Soc. Math. Phys. Eng. Sci., vol. 369, no. 1955, pp. 4558-4576, Nov. 2011.

[2] L. Gagnon, K. Perdue, D. N. Greve, D. Goldenholz, G. Kaskhedikar, and D. A. Boas, "Improved recovery of the hemodynamic response in diffuse optical imaging using short optode separations and state-space modeling," NeuroImage, vol. 56, no. 3, pp. 1362-1371, Jun. 2011.

[3] B. W. Zeff, B. R. White, H. Dehghani, B. L. Schlaggar, and J. P. Culver, "Retinotopic mapping of adult human visual cortex with highdensity diffuse optical tomography," Proc. Natl. Acad. Sci., vol. 104, no. 29, pp. 12169-12174, Jul. 2007.

[4] A. T. Eggebrecht et al., "Mapping distributed brain function and networks with diffuse optical tomography," Nat. Photonics, vol. 8, no. 6, pp. 448-454, Jun. 2014.

[5] D. Chitnis et al., "Functional imaging of the human brain using a modular, fibre-less, high-density diffuse optical tomography system," Biomed. Opt. Express, vol. 7, no. 10, pp. 4275-4288, Oct. 2016.

[6] M. Schweiger and S. Arridge, "The Toast++ software suite for forward and inverse modeling in optical tomography," J. Biomed. Opt., vol. 19, no. 4, pp. 040801-040801, 2014.

[7] S. Lloyd-Fox, A. Blasi, A. Volein, N. Everdell, C. E. Elwell, and M. H. Johnson, "Social Perception in Infancy: A Near Infrared Spectroscopy Study," Child Dev., vol. 80, no. 4, pp. 986-999, 2009. 